\title{
Phenotypic heterogeneity in the stargazin allelic series
}

\author{
Verity A. Letts, ${ }^{1}$ Myoung-Goo Kang, ${ }^{2}$ Connie L. Mahaffey, ${ }^{1}$ Barbara Beyer, ${ }^{1}$ \\ Heather Tenbrink, ${ }^{1}$ Kevin P. Campbell, $^{2}$ Wayne N. Frankel ${ }^{1}$
}

\author{
${ }^{1}$ The Jackson Laboratory, 600 Main Street, Bar Harbor, Maine 04609, USA \\ ${ }^{2}$ The Howard Hughes Medical Institute, Department of Physiology and Biophysics, Department of Neurology, \\ University of Iowa College of Medicine, Iowa City, Iowa 52242, USA
}

Received: 14 January 2003 / Accepted: 4 April 2003

\section{Abstract}

The stargazer mutant mouse is characterized by its ataxic gait, head tossing, and absence seizures. The mutation was identified in the gamma2 subunit gene of the high voltage-dependent calcium channel, Cacng2. Subsequently, two allelic variants of stargazer have arisen, waggler and stargazer 3J. In this study, we have compared these new alleles to the original stargazer allele. All three mutations affect the Cacng2 mRNA levels as they all arise from disruptions within the introns of this gene. Our results show that the mutations cause reduced Cacng2 mRNA and protein levels. Stargazer and waggler mice have the least amount of mRNA and undetectable protein, whereas stargazer $3 \mathrm{~J}$ appears to be the mildest allele, both in terms of the phenotype and protein expression. Electroencephalographic (EEG) analysis confirmed that stargazer has frequent spike-wave discharges (SWDs); the average duration of each discharge burst is 5 seconds and recurs every minute. The waggler allele causes a greater variation in SWD activity depending on the individual mouse, and the stargazer 3J mouse has no SWDs. The preliminary characterization of this heterogeneous allelic series provides a basis to explore more biochemical and physiological parameters relating to the role of the Cacng2 product in calcium channel activity, AMPA receptor localization, and cerebellar disturbances.

The stargazer mutant mouse was originally identified by its ataxic gait and distinctive head-lifting activity (Noebels et al. 1990). The ataxia is first apparent at 14 days of age. Electroencephalographic

Correspondence to: V.A. Letts; E-mail: val@jax.org
(EEG) studies revealed that this mouse has longlasting and frequently recurring absence seizure episodes, characterized by repeated spontaneous spikewave discharge (SWD) activity. This is first detected from around 18 days and continues throughout life without any noticeable impact on the total life span of the mouse.

The stargazer mutation is an early transposon (ETn) insertion in intron 2 of Cacng2, the gene encoding the protein known as stargazin (Letts et al. 1998). By Northern analysis, no mRNA could be detected in total brain preparations, indicating that this mutation clearly affects the message level of the Cacng2 gene. Immunoprecipitation results with antibody directed against a peptide downstream of the ETn insertion revealed that two closely related CACNG subunits, CACNG3 and CACNG4, were present, but no CACNG2 product could be detected (Sharp et al. 2001). Thus, it appears that the stargazer mutant is grossly lacking in any expression of the normal Cacng2 gene.

The initial identification of CACNG2 was based on the similarity of this protein to the voltage-dependent calcium channel (VDCC) gamma subunit, CACNG1, a skeletal muscle protein also having a similar integral membrane structure of four transmembrane-spanning domains and an intracellular amino and carboxy terminus. CACNG1 also has $25 \%$ amino acid identity with CACNG2. Furthermore, in vitro studies of CACNG2 with baby hamster kidney cells suggested that CACNG2 affected the inactivation properties of $\mathrm{Ca}_{\mathrm{v}} 2.1 \quad(\mathrm{P} / \mathrm{Q})$ type VDCC channels, resembling the previously observed inactivation kinetics of the CACNG1 molecule (Letts et al. 1998). In a functional assay with Xenopus laevis oocytes, CACNG2 showed an inhibitory effect on calcium current amplitude when co-expressed with other subunits of the VDCC, namely, CACNA1A or CACNA1B, CACNB3, and CAC- 
NA2D1 (Kang et al. 2001). Brain slice studies further revealed that VDCC peak current density was increased by $45 \%$ in stargazer mice (Zhang et al. 2002).

CACNG2 also contributes to the correct targeting of the $\alpha$-amino-3-hydroxyl-5-methyl-4-isoxazolepropionate (AMPA) receptors to the synapse in granule cells of the cerebellum (Chen et al. 2000). CACNG2 associates with the AMPA receptor to target this molecule to the plasma membrane. Subsequently, this complex associates with the postsynaptic protein PSD-95 and migrates to the synapse. The carboxy terminus of the CACNG2 protein, namely, the threonine-threonine-proline-valine motif, is critical for the binding to PSD-95.

Expression of the Cacng2 product is seen throughout the adult mouse brain, but has not been found in other organs (Sharp et al. 2001). Higher levels are detected in dorsal regions of the brain, including the cerebral cortex, hippocampus, thalamus, olfactory bulbs and cerebellum (Sharp et al. 2001; Klugbauer et al. 2000). However, this defect is not accompanied by any obvious alterations in the brain morphology of these mice, although many biochemical differences have now been detected. Significantly, there are many cerebellar defects in stargazer mutant mice, including the AMPA receptor trafficking defects (Hashimoto et al. 1999; Chen et al. 2000), GABA-associated changes (Thompson et al. 1998; Richardson and Leitch 2002), a decrease in brain-derived neurotrophic factor expression and an impairment of the eyeblink conditioning response /Qiao et al. 1996, 1998). This impairment was also observed in waggler mutants (Bao et al. 1998). Alterations in the cortex and hippocampus have also been detected in stargazer mice, including aberrant neuropeptide $\mathrm{Y}$ expression in the mossy fibers of the hippocampus (Chafetz et al. 1995). The layer V pyramidal neurons of the cerebral cortex had no morphological changes, but did show increased excitability and inward rectification (DiPasquale et al. 1997).

An allelic series of mutations can reveal more subtle changes reflecting the function of the normal gene, especially if some normal transcript of the gene is still evident with some alleles. Since the stargazer mutant mouse was identified, two further alleles, waggler and stargazer 3J, have been independently discovered at The Jackson Laboratory. Both were detected by their ataxic gait, and subsequent complementation analysis confirmed that they were alleles of stargazer. Initial studies of the waggler $\left(s g^{\text {wag }}\right)$ mutation have been described previously (Sweet et al. 1991; Sweet 1993; Letts et al. 1998). The third allele, stargazer $3 \mathrm{~J}\left(s \mathrm{sg}^{3 \mathrm{~J}}\right)$ has only recently been identified. We have compared these two alleles with the original stargazer by looking at the mutations underlying these alleles, measuring the expression of the Cacng2 gene, and studying the absence seizure activity in these mice.

\section{Materials and methods}

Mice. The stargazer (formal symbol, Cacng2 $2^{\text {stg/stg }}$; common symbol, stg/stg), waggler (common symbol, $s^{\text {wag }} /$ stg $^{\text {wag }}$ ), and stargazer 3J (common symbol, stg $^{\text {stg } 3 J} /$ stg $^{\text {stg } 3 J} \mid$ mice arose as spontaneous mutations at The Jackson Laboratory, and, along with the parental and control mice $(\mathrm{A} / \mathrm{J}, \mathrm{MRL} / \mathrm{MpJ} 1 \mathrm{pr}, \mathrm{BALB} / \mathrm{cJ}$, and $\mathrm{C} 57 \mathrm{BL} / 6 \mathrm{~J})$, continue to be maintained at The Jackson Laboratory, Bar Harbor, Maine.

The stargazer mutation arose on the A/J inbred background and has since been crossed onto a B6C3Fe (C57BL/6JxC3HeB/FeJ-a/a) hybrid background, retaining only a small region of $\mathrm{A} / \mathrm{J}$ encompassing the stargazer mutation. The waggler mutation arose on the MRL/MpJ background and, through repeated backcrossing, the waggler allele is now on a primarily C57BL/6J (B6) background. The stargazer 3J mutant allele arose on the BALB/cJ inbred line and is also now maintained on the B6 background.

$\boldsymbol{R T}$-PCR. Brain RNA was prepared with Trizol (Invitrogen) and treated with DNase1 (Promega), under the manufacturer's suggested conditions. RNA 12 $\mu \mathrm{g})$ was transcribed with AMV reverse transcriptase (Promega). PCR was performed under the following conditions: $1 \mathrm{~min}$ at $94^{\circ} \mathrm{C}, 2 \mathrm{~min}$ at $55^{\circ} \mathrm{C}$, and $2 \mathrm{~min}$ at $72^{\circ} \mathrm{C}$, for a total of $25-35$ cycles. The primers were as follows: B2mF (5'-CACGCCACCCACCGGAGAATG-3'); B2mR (5'-GATGCTGATCACATGTCTCG$\left.3^{\prime}\right)$; exon $1 \mathrm{~F}$ (5'-CTCAAAAGCTTGATGACCATC-3'); exon1R (5'-AAGTTCCCTTCGAGGCAG -3'); exon2R (5'-TGCGGTGTCAGCTTCGTAGTC-3'); exon3R (5'ACGAAGAAGGTGCCAGCA-3'); exon4R (5'-ACCATCTCGGCGATGATGAAG-3').

Real-time PCR. RNA was prepared from the brain cortex of the mutant mice and controls with Trizol. Following DNase1 digestion, the RNA concentration and purity was assessed by the Agilent 2100 Bioanalyzer total RNA nano assay. RNA ( $2 \mu \mathrm{g})$ was reverse transcribed with AMV reverse transciptase. The cDNA was diluted one 100-fold, and $1 \mu \mathrm{L}$ was added to Sybr Green PCR mix (Applied Biosystems) with pairs of the following primers; 18SrRNAF (5'-CGGCTACCACATCCAAGGAA-3') and 18SrRNAR (5'-GGGCCTCCGAAAGAGTCCTGTA-3'), Cacng2exon1F (5'-GCTGTTTGATCGAGGTGTTCAA-3') and Cacng2exon1R (5'-CTTGCTGGTTTCATTCTCACTGA-3'), Cacng2exon4F (5'-TACTCCTACGGCTGGTCCTTCTAC-3') and Cacng2exon4R 
(5'-TGTTTGTGGCGGTCGATAAA-3'). The "RT minus" control lacked only the reverse transcriptase enzyme. The PCR reactions were analyzed on an ABI Prism 7700 sequence detector (Perkin Elmer). The PCR amplifications were run in triplicate from two separate reverse transcriptase reactions, each prepared from the cortex of two age-matched animals (between 1 and 2 months) for each strain. Confirmation that the correct size products were amplified was determined by $1.2 \%$ agarose gel electrophoresis.

Western blot analysis. The neuronal calcium channel preparations were prepared as described by Kang et al. (2001) and were resolved by $4-15 \%$ gradient SDS-polyacrylamide gel electrophoresis under reducing conditions ( $2 \%$ beta-mercaptoethanol). The gradient gel was transferred into polyvinylidene difluoride membranes (Millipore), and the blots were probed with affinity-purified antibodies against the subunits of the calcium channel complex at $4^{\circ} \mathrm{C}$ overnight (Kang et al. 2001). After being stained with horseradish peroxidase-conjugated secondary antibodies (Roche Molecular Biochemicals), the blots were developed by enhanced chemiluminescence (ECL) (Supersignal, Pierce) and imaged with an image-capturing system (MultiImage, Alpha Innotech).

Electrode implantation and EEG measurements. Homozygous stg/stg, stgwag $/ \mathrm{stg}^{\text {wag }}$, and $s \mathrm{tg}^{3 \mathrm{~J}} /$ $s t g^{3 J}$ and control heterozygous mice (8-25 weeks of age) were tested for spontaneous spike wave discharge activity. Mice were anesthetized with tribromoethanol (400 $\mathrm{mg} / \mathrm{kg}$ i.p.) and placed in a stereotaxic holder fitted with a mouse incisor bar. Burr holes were drilled $(1 \mathrm{~mm}$ posterior to bregma, 1 $\mathrm{mm}$ lateral to midline) on both sides of the skull. Two teflon-coated bipolar electrodes were implanted at $0.1-0.5 \mathrm{~mm}$ below the dura. Screws were placed at the periphery of the skull to anchor the dental cap. After the mice recovered from surgery, EEG recordings were taken over a 3-day period, for a maximum of $3 \mathrm{~h}$ each day, by using a Grass EEG Model 12 Neurodata Acquisition System and PolyviewPro software program. The parameters for detecting spike wave discharges were described previously (Hosford et al. 1995).

\section{Results}

Comparison of the phenotype of the stargazer allelic series. From observing the effects of three different Cacng2 allelic mutants, stargazer mice appear to be the most grossly affected. In addition to an ataxic gait observed throughout its life, the mouse has a distinctive head-raising motion that becomes more pronounced as the mouse ages. Females are fertile, but males are generally considered infertile, although one or two males have sired single litters from crosses to female stargazer mice.

The waggler mutant also has an ataxic gait, but lacks the head-tossing motion of stargazer. The mouse continues to be ataxic throughout its life. Males and females are fertile, although crosses between homozygous mice often result in only one surviving litter. Both males and females of the stargazer 3J strain are fertile and produce many litters. At 3-4 weeks of age, the affected stargazer 3J homozygotes are readily identifiable by their ataxic gait, but as the mice age and become more sedentary, the ataxia becomes less obvious.

For direct comparative analysis, we attempted to put all three mutations on the same congenic C57BL/6J background, but were thwarted by the stargazer mutation. Attempts to move the stargazer mutation onto an entirely B6 background led to a very reduced birth number of stargazer pups at the N10 backcross, $F_{2}$ intercross generation. The survivors that were born were generally runted and died before weaning. The few that did survive beyond 4 weeks of age continued to live for a normal life span but failed to breed.

\section{Expression profiling of the three allelic mu-} tants. Northern analysis of brain RNA preparations revealed that the wild-type $\mathrm{B} 6$ mice had two major products of 3 and $6-7 \mathrm{~kb}$, and neither of these products could be detected in the stargazer or waggler mutants (Letts et al. 1998; and unpublished data). However, RT-PCR is a more sensitive assay, and amplification of brain RNA by this procedure showed that the stargazer mutants had very reduced levels of normal-sized Cacng2 message. The waggler mutants also had a reduced amount of message encompassing the entire open reading frame. We were able to establish that the mutation in waggler probably lies within the first intron of the Cacng gene. The first exon could be amplified by using specific exon 1 primers, but the amplification products from the first exon to the second, third, or fourth exons were very reduced (Fig. 1). These results indicate that the first intron probably carries a disruption that is affecting normal splicing between the first and second exons (Fig. 2). The intron is more than $100 \mathrm{~kb}$, and we have yet to determine the precise nature of the mutation.

In the stargazer 3J mutants, the Cacng2 message is detectable by Northern analysis. RT-PCR results also showed the normal message and two weaker, higher molecular weight products (Fig. 1). From se- 


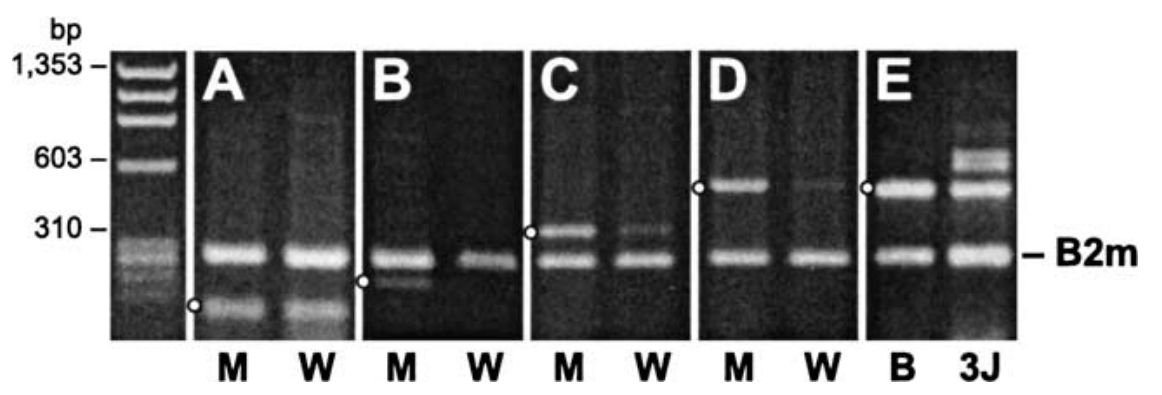

Fig. 1. RT-PCR results from brain RNAs of M, MRL/MpJ; W, stg ${ }^{\text {wag }} /$ stg $^{\text {wag }}$; B, $\mathrm{BALB} / \mathrm{cJ}$; and $3 \mathrm{~J}$, stg ${ }^{3 I} /$ stg $^{3 I}$ mice. Panel A: PCR primers exon1F, exon1R (product size $160 \mathrm{bp}$ ); B, exon1F, exon2R (240 $\mathrm{bp})$; C, exon1F, exon3R (370 bp); D and $\mathrm{E}$, exon $1 \mathrm{~F}$, exon4R (520 bp). The invariant $\mathrm{B} 2 \mathrm{~m}$, beta 2 microglobulin, PCR product $(277 \mathrm{bp}$ ) in every lane serves as a loading control, and the PhiX Hae3-digested DNA marker is shown in the left lane. quencing these higher molecular weight bands, we determined that the stargazer $3 \mathrm{~J}$ mutation was caused by an early transposon (ETn) insertion in the second intron of the Cacng2 gene. We determined that the insert lies at least $2 \mathrm{~kb}$ downstream from the site of the ETn that gave rise to the original stargazer mutation. The larger mRNAs arise from mis-splicing events due to splicing from exon 2 into the long terminal repeat (LTR) of the ETn and back into exon 3 (Fig. 2). Both products splice into the same splice acceptor site of the LTR, but the splice donor varies, giving rise to two products that include 168 or $183 \mathrm{bp}$ of the LTR. Both of these larger messages have inframe insertions and together constitute $26 \%$ of the total Cacng2 product, as determined from densi- tometry scanning of the RT-PCR products run on an agarose gel.

We investigated the Cacng2 message levels in the cortex from the three strains further by real-time PCR. The results in Table 1 are expressed as a percentage of the control C57BL/6J message levels. The two Cacng2 primer pairs were selected to amplify only Cacng2 products. Control experiments confirmed that these primers failed to amplify cDNA from either of the closely related family members, Cacng3 or Cacng4 (results not shown). However, neither of the Cacng2 primer amplification products spanned an intron. An RT minus control, lacking only the reverse transcriptase enzyme, was used to measure contaminating DNA levels, which proved

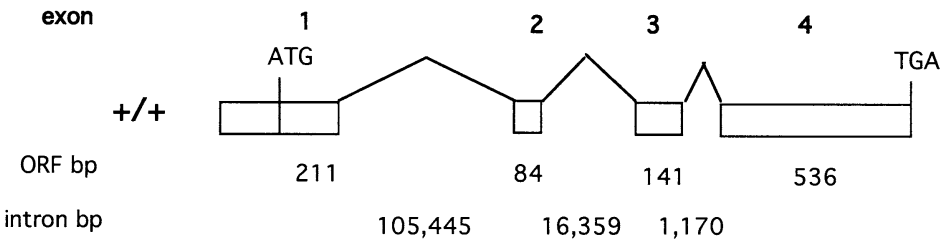

stargazer

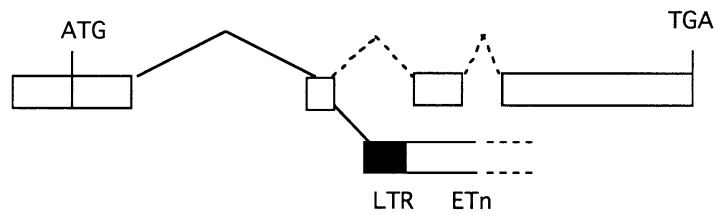

waggler

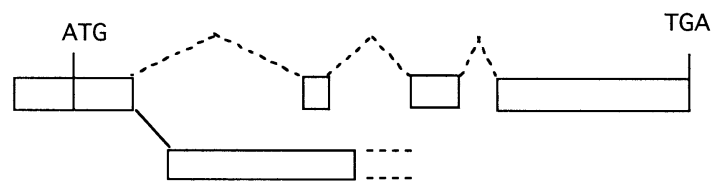

stargazer3J

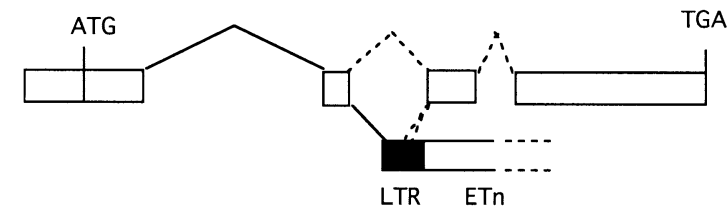

Fig. 2. Schematic illustration of the three spontaneous mutations in the Cacng2 gene. 
Table 1. Real-time PCR results showing Cacng2 exon1 and exon4 expression. Delta $C_{T}$, difference in threshold cycle value for mutants from the $\mathrm{C57} \mathrm{BL} / 6 \mathrm{~J}$ control; s.d., standard deviation. All $\mathrm{C}_{\mathrm{T}}$ values were initially standardized against their endogenous control, 18S rRNA. The "average \% of C57BL/6J" is determined relative to the $\mathrm{C} 57 \mathrm{BL} / 6 \mathrm{~J}$ set at $100 \%$.

\begin{tabular}{lcccccccc}
\hline & \multicolumn{3}{c}{ Cacng2 Exon1 } & & \multicolumn{3}{c}{ Cacng2 Exon4 } \\
\cline { 2 - 3 } & Average delta $C_{T}$ & s.d. & Average \% of C57BL/6I & & Average delta $C_{T}$ & s.d. & Average \% of C57BL/6J \\
\hline stargazer & 0.86 & 0.40 & 56 & 25 & & 5.09 & 0.52 & 3 \\
waggler & 2.00 & 0.74 & 50 & & 1.94 & 0.86 & 3.5 \\
stargazer 3J & 0.99 & 0.40 & & 50 & 0.58 & 28 \\
\hline
\end{tabular}

to be negligible in all samples. $18 \mathrm{~S}$ ribosomal RNA was used as the internal control.

To compare the Cacng2 mRNA levels, we examined amplification products from exon 1 and exon4. Amplification with exon 1 primers revealed that stargazer and stargazer $3 \mathrm{~J}$ mutants both had $50-56 \%$ of the B6 level of exon 1 cDNA, and waggler mutants had $25 \%$. We predict that the waggler mutation lies within intron 1, and expression of the first exon was reduced compared with the two other alleles with mutations in intron 2. Primers to exon 4 amplify a product from the full-length Cacng2 message only. Noticeably, stargazer mutants had only $3 \%$ of the normal message, waggler had $3.5 \%$, and stargazer $3 \mathrm{~J}$ mutants had $28 \%$ of the normal message. Included in this last figure are the two aberrant products that together constitute one quarter of the total Cacng2 message in stargazer 3J mutants. The waggler preparations showed significantly higher standard deviations compared with stargazer and stargazer 3J, but the average percentage of Cacng2 message was similar to stargazer.

No CACNG2 product was detected in the total brain preparation from the stargazer mouse by Western blot analysis with an antibody that was generated against the common peptide of the CACNG2 and CACNG3 proteins, downstream of the disrupted sites of all three alleles in the Cacng2 gene

\section{B6 stg stg3J wag}

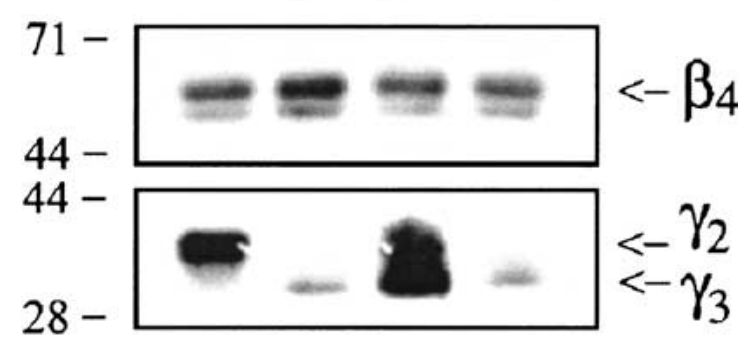

Fig. 3. CACNG2 protein expression in the brain of the stargazer alleles. Comparison of CACNG2 protein expression in $\mathrm{C} 57 \mathrm{BL} / 6 \mathrm{~J}$ (B6), stargazer (stg), stargazer 3J (stg3J), and waggler (wag). Each lane was loaded with 100 $\mathrm{mg}$ of protein. Arrows show the CACNB4 ( $\beta 4)$, CACNG2 $(\gamma 2)$, and CACNG3 $(\gamma 3)$ products.
(Fig. 3). However, CACNG3 and CACNB4 proteins were still present. The waggler CACNG2 protein was also missing, and only the stargazer 3J brain preparation had detectable levels of the CACNG2 protein. No higher molecular weight proteins corresponding to the aberrant mRNAs were observed, but a significant increase in the CACNG3 protein band was observed. We did not detect any increase in Cacng3 mRNA expression in stargazer 3J mutants, so this increase in the $30-\mathrm{kDa}$ band is either due to an increased post-translational CACNG3 expression and/or protein stability or could include products translated from the aberrant Cacng2 mRNA products.

\section{Electroencephalographic (EEG) recordings} show distinct differences. One striking feature of stargazer is the spontaneous recurrent episodes of absence seizure activity. Of all the mouse absence seizure mutations studied so far, the stargazer appeared to be the most severely affected in terms of the duration of each seizure episode, and the frequency of recurrence of these seizure episodes (Noebels et al. 1990). The spike-wave discharge (SWD) bursts from stargazer were characteristically between 5 and $7 \mathrm{~Hz}$ and lasted for several seconds from very short episodes of $1 \mathrm{~s}$ to much longer activities lasting $30 \mathrm{~s}$ or more. These seizure episodes recurred many times, and the mouse was awake but unmoving during these episodes, and these seizures continued throughout the life of the mouse. The seizures were abolished by intraperitoneal injection of the anti-absence seizure drug, ethosuximide (150 $\mathrm{mg} / \mathrm{kg})$.

The waggler mouse also had absence seizures, with spike-wave discharges of between 5 and $7 \mathrm{~Hz}$. The duration and recurrence of these episodes varied from animal to animal, but from the combined analysis of eight waggler mice, the seizure episodes lasted on average one second and recurred every 15 minutes. However, included within these eight mice there was one that had no seizures over the 9-h recording period, and one that had seizures almost as frequently as stargazer mutants, revealing the 

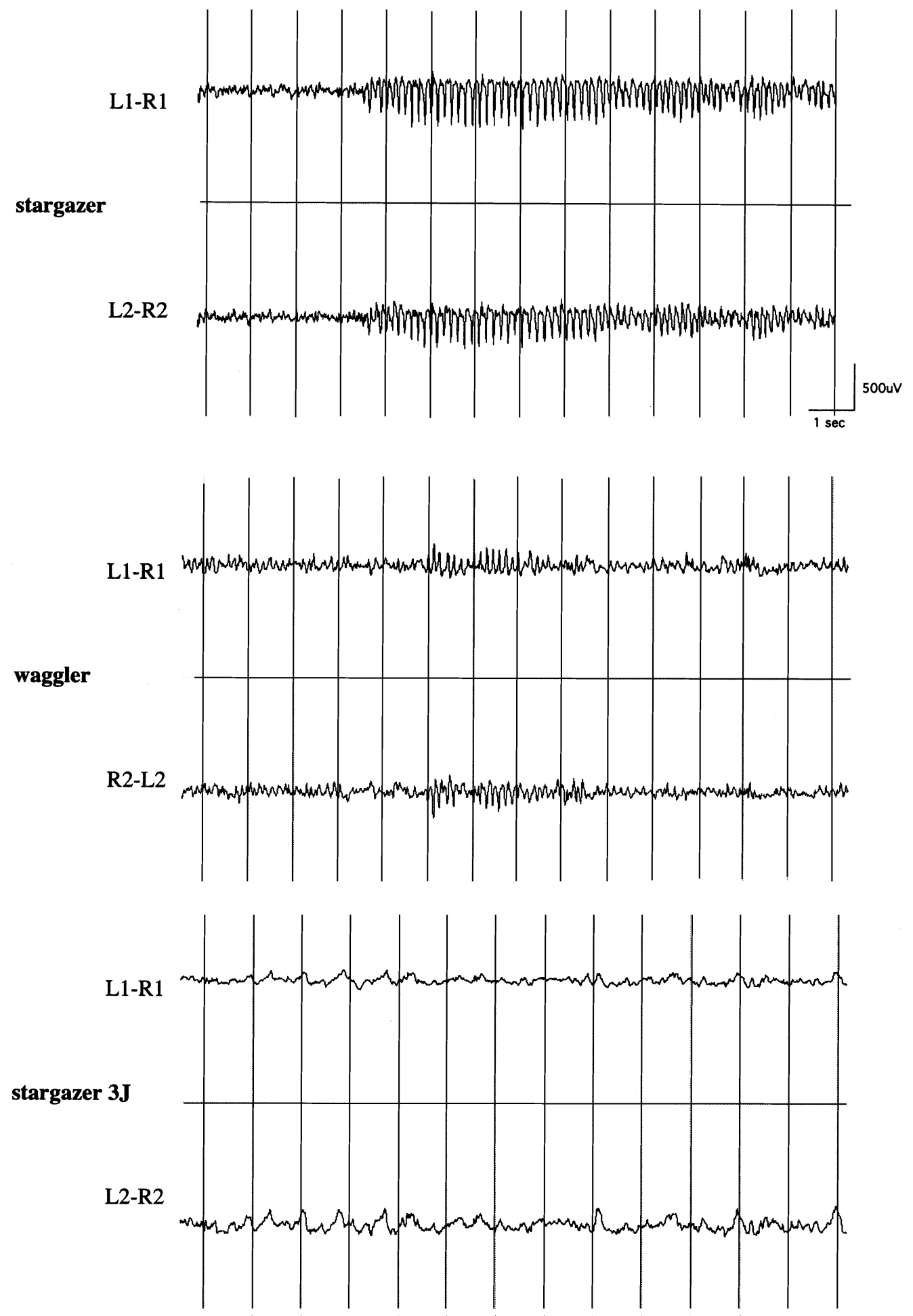

Fig. 4. Typical EEG tracings from stargazer, waggler and stargazer $3 \mathrm{~J}$ mice. L1-R1, recordings comparing signal from the left cortical electrode to the right electrode. L2-R2, recordings comparing output from the second electrode of the left and right side of the bipolar electrodes. The episode of SWD bursts in stargazer starts after $4 \mathrm{~s}$ and continue to the end, and the SWDs in waggler are observed between 5 and $7 \mathrm{~s}$ of this tracing.

variable expression of this particular phenotype. The stargazer 3J mouse failed to show any seizure episodes throughout the recordings. A typical EEG recording for each allele is shown in Fig. 4. The average duration of each SWD burst and recurrence of SWD bursts illustrates the striking difference between stargazer and stargazer 3J mice (Table 2).

\section{Discussion}

The founding member of this allelic series described here is the stargazer mutant mouse. Of the three allelic variants, stargazer appears to be the most severely affected for seizure activity and mobility. The waggler mutants have a similar ataxic disorder to 
Table 2. Comparative seizure duration and recurrence in the three mouse mutants

\begin{tabular}{lccc}
\hline $\begin{array}{l}\text { Mouse } \\
\text { mutation }\end{array}$ & $\begin{array}{c}\text { Average seizure } \\
\text { duration (secs) }\end{array}$ & $\begin{array}{c}\text { Average seizure } \\
\text { recurrence (mins) }\end{array}$ & $\begin{array}{c}\text { No. of mice with SWDs/ } \\
\text { no. of mice tested }\end{array}$ \\
\hline stargazer & 5 & 1 & $8 / 8$ \\
waggler & 1 & 15 & $7 / 8$ \\
stargazer 3J & 0 & 0 & $0 / 7$ \\
\hline
\end{tabular}

stargazer, but lack the head movements, and the stargazer 3J mutants are the most mildly affected. Interestingly, none of the three alleles have missense or nonsense mutations within the open reading frame of the Cacng2 gene; instead, all appear to arise from disruptions within the introns of this gene. This type of mutation is unlikely to generate complete nulls, and even in stargazer mice we can detect very low levels of normal-sized mRNA in sensitive RT-PCR and real-time PCR assays.

Mutations arising from intron disruptions also result in variable phenotypic effects as individual cells will not necessarily be consistent in terms of their ability to circumnavigate the disruption to produce normally spliced messenger RNA. Thus, although looking at gross morphological changes may be reliably reproducible, more detailed investigations may be confounded by this heterogeneity of expression. This point is illustrated by the waggler mouse, which shows a variable profile of SWDs and a significant range in Cacng2 mRNA levels. Finally, from the stargazer 3J allele, we can determine that Cacng2 message levels as low as $28 \%$ of the B6 control are still sufficient to suppress seizures.

The differing severity of the mutations in the stargazer allelic series provides the opportunity to study the correlation between loss of Cacng2 expression and seizure onset in a relatively quantitative manner. By combining these mutations, it will be possible to determine whether there is a quantifiable threshold of gamma2 expression below which seizures are observed. For instance, stg/stg ${ }^{3 I}$ compound heterozygous mice should retain about $16 \%$ of the B6 levels of Cacng2 message. Future studies can also exploit the variation in the waggler phenotype to confirm if there is a direct correlation between the frequency and duration of SWD bursts with Cacng2 mRNA levels in this mutation.

The Cacng gene family is represented by eight members, seven of which are expressed in the brain (Chu et al. 2001). It appears that gamma2 is the most highly expressed, and thus mutations in this gene are more likely to have a direct impact on the mouse phenotype. To date, only mutations in the Cacng2 gene have been detected spontaneously owing to their ataxic gait.
One explanation for the lack of mutants with defects in other Cacng genes may be that mutations in these brain-related gamma family members may not give rise to obvious movement disorders and consequently have never been picked up by deviant screens. Furthermore, there may be compensation within the Cacng gene family. Although there appears to be no obvious compensation in vivo for the absence of CACNG2 by CACNG3 and CACNG4 proteins (Sharp et al. 2001), there is in vitro evidence for the potential of functional compensation. In studies with Xenopus oocytes, CACNG2 had an inhibitory effect on calcium current amplitude when co-expressed with CACNA1A or CACNA1B, CACNB3, and CACNA2D, and it was found that CACNG1 could functionally replace CACNG2, even though CACNG1 expression is normally confined to skeletal muscle (Kang et al. 2001). Thus, there is something intrinsic to the common structure of these CACNG subunits that allows them to compensate for each other, although the heterogeneity of the gamma family suggests that each gamma molecule also retains more specialized functions. The relatively high expression of CACNG2 may therefore mask any phenotypic effects of mutations in other brainspecific CACNG genes.

In addition to the increased VDCC peak current activity in stargazer and tottering mutants, there is also an increase in the peak current density of lowvoltage calcium channels in stargazer, tottering, and lethargic mice (Zhang et al. 2002). These $\mathrm{Ca}_{\mathrm{v}} 3$ or Ttype channels are directly responsible for the postinhibitory rebound burst firing in thalamocortical relay cells. These neurons, together with the thalamic reticular nucleus and the cortical pyramidal cells, form the thalamocortical loop that is believed to generate the spike-wave discharges when the normal balance between excitatory and inhibitory discharges within this circuit is perturbed /Snead 1995; McCormick and Contreras 2001; Crunelli and Leresche 2002). Further studies on the other alleles of stargazer may contribute to understanding the function of CACNG2, especially in relation to its role in preventing the occurrence of absence seizures. 


\section{Acknowledgments}

We thank Drs. Susan Ackerman and Muriel Davisson for critical reviews of this manuscript. This work was supported by a grant from the NIH NS32801 to Verity A. Letts. Myoung-Goo. Kang was partly funded by an Epilepsy Foundation Predoctoral Fellowship, and Kevin P. Campbell is an Investigator of the Howard Hughes Medical Institute.

\section{References}

1. Bao S, Chen L, Qiao X, Knusel B, Thompson RF (1998) Impaired eye-blink conditioning in waggler, a mutant mouse with cerebellar BDNF deficiency. Learn Mem 5, 355-364

2. Chafetz RS, Nahm WK, Noebels JL (1995) Aberrant expression of neuropeptide $\mathrm{Y}$ in hippocampal mossy fibers in the absence of local cell injury following the onset of spike-wave synchronization. Brain Res Mol Brain Res 31, 111-121

3. Chen L, Chetkovich DM, Petralia RS, Sweeney NT, Kawasaki Y et al. (2000) Stargazin regulates synaptic targeting of AMPA receptors by two distinct mechanisms. Nature 408, 936-943

4. Chu PJ, Robertson HM, Best PM (2001) Calcium channel gamma subunits provide insights into the evolution of this gene family. Gene 280, 37-48

5. Crunelli V, Leresche N (2002) Childhood absence epilepsy: genes, channels, neurons and networks. Nat Rev Neurosci 3, 371-382

6. DiPasquale E, Keegan KD, Noebels JL (1997) Increased excitability and inward rectification in layer $\mathrm{V}$ cortical pyramidal neurons in the epileptic mutant mouse Stargazer. J Neurophysiol 77, 621-631

7. Hashimoto K, Fukaya M, Qiao X, Sakimura K, Watanabe $M$ et al. (1999) Impairment of AMPA receptor function in cerebellar granule cells of ataxic mutant mouse stargazer. J Neurosci 19, 6027-6036

8. Hosford DA, Lin FH, Kraemer DL, Cao Z, Wang Y et al. (1995) Neural network of structures in which GABA $_{B}$ receptors regulate absence seizures in the lethargic $(\mathrm{lh} /$ lh) mouse model. J Neurosci 15, 7367-7376

9. Kang MG, Chen CC, Felix R, Letts VA, Frankel WN et al. (2001) Biochemical and biophysical evidence for gamma 2 subunit association with neuronal voltageactivated $\mathrm{Ca} 2+$ channels. J Biol Chem 276, 32917-32924
10. Klugbauer N, Dai S, Specht V, Lacinova L, Marais E et al. (2000) A family of gamma-like calcium channel subunits. FEBS Lett 470, 189-197

11. Letts VA, Felix R, Biddlecome GH, Arikkath J, Mahaffey CL et al. (1998) The mouse stargazer gene encodes a neuronal $\mathrm{Ca} 2+$-channel gamma subunit. Nat Genet 19, 340-347

12. McCormick DA, Contreras D (2001) On the cellular and network bases of epileptic seizures. Annu Rev Physiol 63, 815-846

13. Noebels JL, Qiao X, Bronson RT, Spencer C, Davisson MT (1990) Stargazer: a new neurological mutant on Chromosome 15 in the mouse with prolonged cortical seizures. Epilepsy Res 7, 129-135

14. Qiao X, Hefti F, Knusel B, Noebels JL (1996) Selective failure of brain-derived neurotrophic factor mRNA expression in the cerebellum of stargazer, a mutant mouse with ataxia. J Neurosci 16, 640-648

15. Qiao X, Chen L, Gao H, Bao S, Hefti F (1998) Cerebellar brain-derived neurotrophic factor-TrkB defect associated with impairment of eyeblink conditioning in Stargazer mutant mice. J Neurosci 18, 69906999

16. Richardson CA, Leitch B (2002) Cerebellar Golgi, Purkinje, and basket cells have reduced gamma-aminobutyric acid immunoreactivity in stargazer mutant mice. J Comp Neurol 453, 85-99

17. Sharp AH, Black JL 3rd, Dubel SJ, Sundarraj S, Shen JP et al. (2001) Biochemical and anatomical evidence for specialized voltage-dependent calcium channel gamma isoform expression in the epileptic and ataxic mouse, stargazer. Neuroscience 105, 599-617

18. Snead OC (1995) Basic mechanisms of generalized absence seizures Ann Neurol 37, 146-157

19. Sweet HO (1993) Waggler and stargazer are allelic. Mouse Genome 91, 312-313

20. Sweet HO, Bronson RT, Cook S, Spencer C, Davisson MT (1991) Research News. 1. Waggler (wag). Mouse Genome 89, 552

21. Thompson CL, Tehrani MH, Barnes EM, Stephenson FA (1998) Decreased expression of GABAA receptor alpha 6 and beta 3 subunits in stargazer mutant mice: a possible role for brain-derived neurotrophic factor in the regulation of cerebellar $\mathrm{GABA}_{\mathrm{A}}$ receptor expression? Brain Res Mol Brain Res 60, 282-290

22. Zhang Y, Mori M, Burgess DL, Noebels JL (2002) Mutations in high-voltage-activated calcium channel genes stimulate low-voltage-activated currents in mouse thalamic relay neurons. J Neurosci 22, 6362-6371 